\title{
Interval Type-2 Fuzzy Logic System for Dynamic Spectrum Access in Cognitive Radio
}

\author{
Mahesh V. Lakhekar \\ Department of Electronics and Telecommunication \\ Engineering \\ M.G.M's College of Engineering, Nanded
}

\author{
Shirish L. Kotgire \\ Department of Electronics and \\ Telecommunication Engineering \\ M.G.M's College of Engineering, Nanded
}

\begin{abstract}
The current scenario has shown that, with the conventional spectrum access approach, the radio spectrum allocated to primary (licensed) users is hugely underutilized. While many spectrum methods have been proposed to utilize spectrum efficient manner, the spectrum access opportunistic way is happen to the most practical approach to attain near-optimal spectrum utilization by permitting secondary (unlicensed) users to sense and access available spectrum opportunistically. In this paper, we present decision making scheme in cognitive radio based on Interval type-2 fuzzy logic system. Here, classical type-1 and Interval type-2 fuzzy logic system has been compared in terms of possibility of spectrum access by the secondary user with effective and seamless communication between cognitive radio and primary user. The proposed fuzzy inference system has three input parameters such as spectrum utilization efficiency, degree of mobility and distance to primary user of cognitive radio, along with output parameter as the possibility of accessing the spectrum for secondary user based on linguistic knowledge of 27 rules. This paper mainly deals with design of decision making scheme using Interval type-2 fuzzy logic for minimizing the effect of uncertainty produced by the measurement and environmental noise. Simulation result shows significant enhancement in dynamic spectrum allocation for secondary user with higher probability conditions.
\end{abstract}

\section{Keywords}

Cognitive Radio, Type-1 fuzzy logic, Interval Type-2 Fuzzy logic System, Spectrum Access.

\section{INTRODUCTION}

The electromagnetic radio spectrum as a natural resource is utilized by transmitters and receivers in communication network, which is regulated by government agencies. According to statics of the Federal Communications Commission (FCC), that the conventional fixed spectrum allocation strategies have issues of underutilization in currently deployed frequency bands. In many bands, spectrum access is a more important task than physical insufficiency of spectrum, in major part due to legacy command-and-control regulation that limits the capability of spectrum users to acquire such access. In fact, if we examine portions of the radio spectrum used in the urban areas, then we find that several frequency bands in the spectrum are regularly vacant in most of the time and other frequency bands are partially occupied, while the remaining frequency bands are heavily utilized. The efficient use of existing spectrum is become most critical problem as growing demand of the radio spectrum.

The solution to this problem provided by Cognitive radio (CR) as the means to promote the efficient use of the spectrum by exploiting the existence of spectrum holes. It is defined as a software-defined radio, can change its transmitter parameters based on interaction with its operational electromagnetic environment, which was proposed by Joseph Mitola for effective utilization of spectrum [1]. The major functions of the CR are spectrum sensing, spectrum mobility, spectrum allocation and optimization of the resources. Spectrum sensing means to determine spectrum availability and the presence of the licensed user. Spectrum management is the task of capturing the best accessible spectrum to meet user communication requirements [2]. Spectrum allocation is refers to providing a fair spectrum scheduling method among the users, which is the major challenge in the open spectrum usage. Spectrum mobility is defined as the process where the cognitive user exchanges its frequency of operation. By using these functions a model is planned where an unlicensed user can employ available licensed spectrum in dynamic method depending on the possibility of access based on external parameters.

In recent development, many ways are being found for effective spectrum utilization and seamless communication between CR and licensed user or primary user (PU). CR is intelligent technique for detecting an unused portion of the spectrum or white space can be utilized by PU. If unused slots are identified, then can be accessed by secondary users (SU) with offered condition is that, SU need to vacant the frequency bands as quickly as possible when again required by $\mathrm{PU}$ in order to avoid interference. In resource management scheme across communication networks required more efficient use of distributed resources as a radio spectrum. The decision making is a challenging task in resource management of $\mathrm{CR}$, because of increased dimensionality and the complex operational environment. As a result of differing requirements and restrictions an engineer compromise need to be sought, specifically for the decision making process. In the complex environment with compressed time scale, optimal solution cannot be found but design required well enough solutions, therefore new design techniques are required in software defined radio. The modern CR heavily based on the knowledge of operational environment for decision making purpose. Here, environment awareness techniques used for gathering information on the current resource use and their state. For obtaining reliable information in dynamic and uncertain environment is difficult task in future CR. The characteristics of future CR's keep in mind, the capabilities of fuzzy logic offer good potential to be utilized in resource management, because fuzzy logic is an attractive technique particularly in case where target problems are complicated to model with conventional mathematical methods, but these problems are at the same time solve through human knowledge. The decision making problems in CR's has more dimensionality and the unpredictable 
operational environment. Therefore, fuzzy logic tool well suited for multidimensional decision making problems [3]. The decision making fuzzy inference system achievable by expert knowledge based learning, which enables efficient inclusion of incomplete information. The flexibility provided by intelligent decision making architecture for dynamic and complex distributed communication network. An interesting survey on usage of fuzzy logic technique in telecommunication field is reported by Gosh et al. [4]. Fuzzy logic which is multi valued logic that permits transitional value to define such as yes/no, High/low. Baldo and Zorzi [5] proposed the distributed cognitive network access scheme with the aim of providing the best quality of service (QoS) with respect to both radio link and core networking performance. The knowledge of service quality experienced by active connection is shared and prospective user employ fuzzy logic technique to process cross layer communication. Matinmikko et al. [6] presented a fuzzy combining scheme for cooperative spectrum sensing in CR system, which is capable of handling multidimensional, contradictory and generally non-predictable in decision making problem. Kaur et al. [7] suggested two level hierarchical fuzzy scheme for adaptive bandwidth allotment is planned where one system evaluate the access latency based on analytical model and the second system allocate the bandwidth to secondary user. Lee and Ly [8] introduced a novel approach using fuzzy logic system to control spectrum access based on three descriptors such as spectrum utilization efficiency, its degree of mobility and its distance to the PU with 27 rule base. In recent CR, Koroupi et al. [9] introduce an innovative approach for making fair assignment of available spectrum to unlicensed user in smart grid communication network. The spectrum assignment algorithm based on fuzzy logic ant colony system as an intelligent bio inspired technique. Afterwards, Subhedar and Birajdar [10] compare two different fuzzy inference system models i.e. Mamdani and Sugeno systems to compute spectrum access decision based on the secondary user parameters first one is signal strength, second is distance to primary user, third spectrum utilization efficiency and last one is degree of mobility with 81 fuzzy rules are used to obtain the output of proposed system as the possibilities of allotment of white spaces to secondary users. From this concise survey, we stated that, fuzzy logic system is capable of making real time decision to decide the suitable secondary user which will use the available band. In this paper, the decision making scheme is formulated on the basis of interval type-2 fuzzy logic system (IT2FLS) which has three inputs and one output for dynamic spectrum allocation in CR. This decision making scheme mainly depends on 27 fuzzy IF-THEN rules design on linguistic knowledge of complex environment in communication network. The concept of IT2FS is an extension of the well known ordinary fuzzy set, suggested by Zadeh (1975). This is more adaptive and they can realize more complex input-output relationship which cannot be achieved by type-1 fuzzy logic system. The control surface of IT2FS is smoother than its type-1 counterpart, specifically in the steady state condition. It also useful especially when there is higher degree of uncertainty occurred in operating environment, means difficult to determine exact value of available spectrum in frequency band in specific time along with geographical location as a measurement uncertainty. The rest of this paper is organized in following manner. The Section II get commence the fundamentals of CR with their elements. Opportunistic spectrum access using IT2FLS based on experiences from a group of network experts is proposed in Section III. In Section IV and $V^{\text {th }}$ section discuss Simulation results, Conclusions and Future works.

\section{PRELIMINARIES}

The term CR originated from the word 'cognitive' which means process of acquiring knowledge by the use of reasoning, intuition or perception. CR is a software defined radio system which adapts to the operating conditions of the environment by investigating, observing and learning. It is a smart technology which scans the radio spectrum and look for white spaces in it, which enables the unlicensed user to utilize the licensed bands without causing any significant interference to the licensed user [1]. The licensed user is also called as primary user (PU), while users which are having no rights to access the licensed bands are known as secondary users (SU). A general cognitive cycle as depicted in Fig.1 for characterizing the operations of CRs in [2]. According to the definition, CRs has the abilities to obtain knowledge, regulate according to the information, and gain knowledge from results.

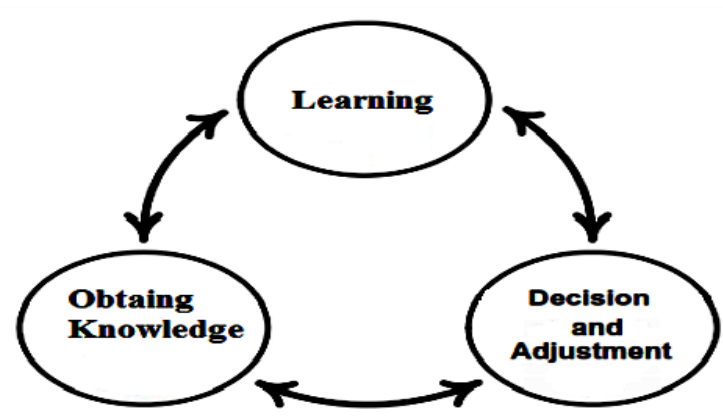

Fig 1: General cognitive cycle

The bar chart represents in Fig.2 various frequency bands accessed by multiple licensed user or primary users in traditional communication technique. The allocated spectrum has not make use of properly, it differ with instance, occurrence and geological locations. Thus to avoid spectrum scarcity and unutilized frequency bands, a new communication technique as $\mathrm{CR}$ and dynamic spectrum access is introduced.

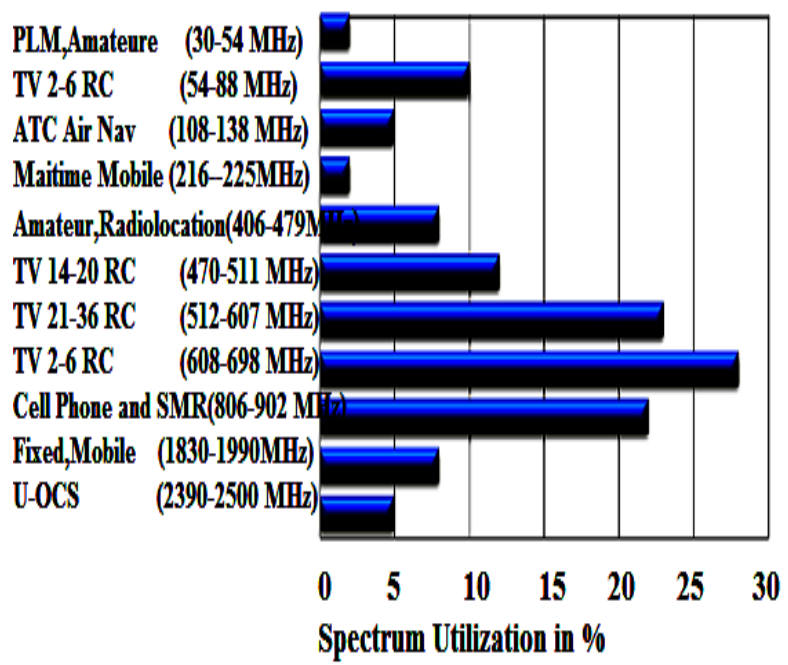

Fig 2: Spectrum Utilization in different band

\subsection{CR Characteristics}

It has two main characteristics which help in achieving this goal, are described as follows [2]: 
Cognitive capability: In real-time interaction with the radio environment, the portions of the spectrum those are vacant at a specific time or location can be identified and CR enables the usage of temporally unused spectrum, referred to as spectrum hole or white space. Thus, the best spectrum can be chosen, shared with other users, and utilized without interference with the licensed.

Reconfigurability: A CR can be programmed to transmit and receive information on a variety of frequencies, and apply diverse access technologies hold up by its hardware design. Due to this ability, the best spectrum band and the good number of suitable operating parameters can be chosen and reconfigured.

In CR's network, fundamental terms are specified and a functional explanation given below.

Primary user: A user is said to be primary, when it has authorized right to access the licensed band, then it is also known as licensed user.

Secondary user: A user is said to be secondary, when it has no permission to use the licensed spectrum, then it is also known as unlicensed user. It detects the radio spectrum and searches for the un-utilized portions of the radio spectrum. It uses this un-utilized spectrum to transmit it signals without affecting the primary user.

White spaces: A vacant portion of the licensed spectrum is known as white space.

It provides opportunity for other secondary users to access it with the help of CR technology. One of the main objectives of CR is to explore for white spaces, which is also known as spectrum holes. The development of $\mathrm{CR}$ techniques can result in new and more efficient ways of interference management. Individual techniques developed in the telecommunication research, e.g. smart antennas and power control, offer a good solution for interference suppression and can be further developed for the needs of CRs. Efficient utilization can be improved by allowing a SU to utilize a licensed band when PU is not available. PU has greater priority to use a particular spectrum and SU has lower priority, to use the spectrum without giving inference to PU. The effective utilization of spectrum carried out in four steps are described as follows [2]:

Spectrum Sensing: In first step is to sense the radio environment and search for white spaces in it, without causing interference to the primary network.

Spectrum Management: It is the task of acquiring the best accessible spectrum to meet user communication requirements.

Spectrum Mobility: The basic idea of CR networks is that the unlicensed users to share the licensed spectrum without interfering with the transmission of other licensed users. In case this band is found to be occupied by a PU, then CR user moves to another spectrum hole to avoid interference, which is referred to as spectrum mobility.

Spectrum Sharing: Sharing is the major challenge in the radio spectrum usage. It provides a fair spectrum scheduling method among the users.

This approach for improving efficiency and increased collaboration between networks and terminals are likely to yield benefits in the usage of spectrum.

The decision making for resource management in CRs is mainly depends on the knowledge of the operational radio environment. The status of radio environment is based on the present resource utilization and state of nature. The accumulated information related to the current spectrum use is uncertain in nature, so that formulation of decision making process in mathematical equation format is impossible. Therefore, fuzzy logic is proposed to employ as generic knowledge base for efficient utilization of available spectrum band. Due to fuzzy logic based decision making, CR's should capable to understand the various service requirements and use artificial intelligence techniques to adapt the configuration of the system in time variant operational environment.

\section{INTERVAL TYPE-2 FUZZY LOGIC SYSTEM (IT2FLS)}

The concept of type-2 fuzzy set (T2FSs) was initially presented as an extension of ordinary fuzzy set that is type-1 fuzzy set (T1FSs). The T1FSs have some demerits to formulate and reduce the effects of uncertainties. As a result, T2FS was developed as a powerful alternative method for addressing these issues (Zadeh, 1974), but main disadvantage of the T2FSs is computational burden. This is because of the type-reducer computations [3]. Therefore, using IT2FLS computations reduces to reasonable amount that makes the implementation of IT2FLS easy. IT2FLS as a special case of T2FSs are now the most widely used for their reduced computational burden. The fuzzy membership function demonstrated in Fig.3, which is bounded from up and down with two T1 fuzzy membership function that are called upper membership function (UMF) and lower membership function (LMF), respectively. The region between UMF and LMF is called footprint of uncertainty.

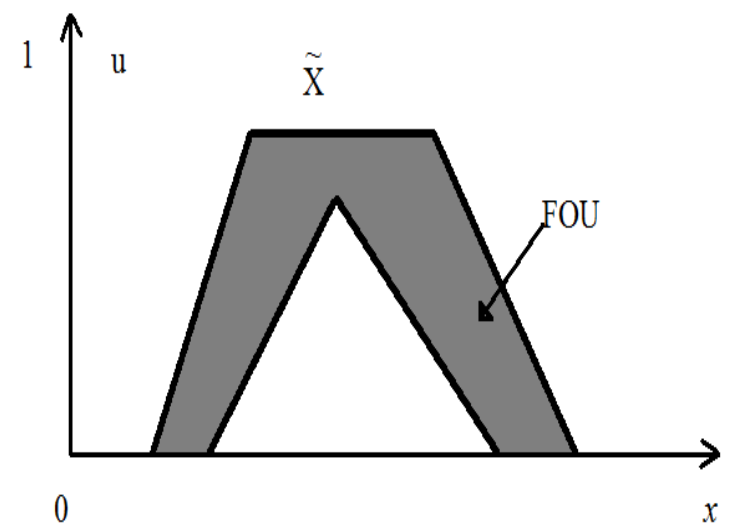

Fig 3: IT2FLS Membership function

The general structure of IT2FLSs is illustrated in Fig. 4, which contains the following five components: fuzzifier, rule base, fuzzy inference engine, type-reducer, and defuzzifier. It is seen that the structure is almost similar to the structure of T1FLSs. Here, the major difference is that at least one of the FSs in the rule base is an IT2FLS. Therefore, the outputs of the inference engine are IT2FLSs and a type reducer is needed in order to convert them into a T1FS. Then, the T1FSs is defuzzified into a crisp number as the output of the IT2FLS. The systematic design procedure of the IT2FLS is described in detail first, then, we propound the proposed decision making model for generating control surface in CR. 


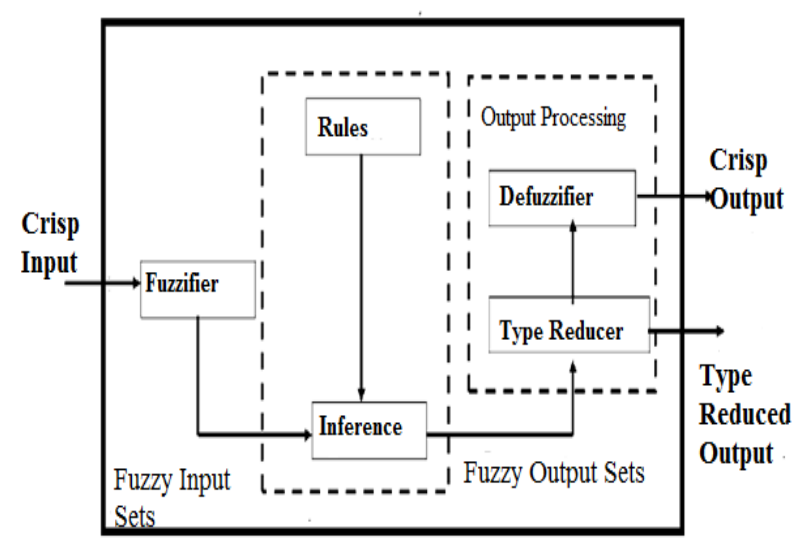

Fig 4: General Representation of an IT2FLS.

In practice, the computations in an IT2FLS can be significantly simplified. Consider the rule base of an IT2FLS consisting of $\mathrm{N}$ rules assuming the following form:

$\mathrm{R}^{n}: I F x_{1}$ is $\tilde{X}_{1}^{n}$ and ........and $x_{I}$ is $\tilde{X}_{I}^{n}$, THEN yis $Y^{n} \quad n=1,2, \ldots \ldots, N$ Where, $\tilde{X}_{i}^{n}(\mathrm{i}=1, \ldots \ldots \ldots, \mathrm{I})$ are IT2FLSs, a $Y^{n}=\left[\underline{y}^{n}, \bar{y}^{n}\right]$ is an interval, which can be understood as the centroid of a consequent IT2FLS, or the simplest TSK model, for its simplicity. In many applications we use $y^{n}=\bar{y}^{n} \quad$ i.e., each rule consequent is a crisp number. Suppose the input vector is $x^{\prime}=\left(x_{1}^{\prime}, x_{2}^{\prime}, \ldots \ldots \ldots . ., x_{I}^{\prime}\right)$ and typical computations in an IT2 FLS involve the following steps:

- Calculate the membership function of $x_{i}^{\prime}$ on each

$$
X_{i}^{n},\left[\mu \underline{X}_{i}^{n}\left(x_{i}^{\prime}\right), \mu \bar{X}_{i}^{n}\left(x_{i}^{\prime}\right)\right]
$$

- $\quad$ Determine the firing interval of the $n^{\text {th }}$ rule, $F^{n}\left(x^{\prime}\right)$ :

$$
\begin{aligned}
& F^{n}\left(x^{\prime}\right)=\left[\mu \underline{X}_{1}^{n}\left(x_{1}^{\prime}\right) \times \ldots \ldots . . . \times \mu \underline{X}_{I}^{n}\left(x_{I}^{\prime}\right), \mu \bar{X}_{1}^{n}\left(x_{1}^{\prime}\right) \times \ldots \ldots . .\right. \\
& \left.\times \mu \bar{X}_{I}^{n}\left(x_{I}^{\prime}\right),\right] \equiv\left[\underline{f}^{n}, \bar{f}^{n}\right], \quad n=1,2,3, \ldots N
\end{aligned}
$$

Note that the minimum, instead of the product, can be used in Eq. (1)

- Perform type-reduction to combine $F^{n}\left(x^{\prime}\right)$ and the related rule consequents. There are many such methods. The most commonly used one is the center-of-sets type-reducer:

$$
Y_{\operatorname{COS}}\left(x^{\prime}\right)=\bigcup_{\substack{f^{n} F^{n}\left(x^{\prime}\right) \\ y^{n} \in Y^{n}}} \frac{\sum_{n=1}^{N} f^{n} y^{n}}{\sum_{n=1}^{N} f^{n}}=\left[y_{l}, y_{r}\right]
$$

It has been show that:

$$
y_{l}=\min _{k \in[1, N-1]} \frac{\sum_{n=1}^{k} \bar{f}^{n} \underline{y}^{n}+\sum_{n=k+1}^{N} \underline{f}^{n} \underline{y}^{n}}{\sum_{n=1}^{k} \bar{f}^{n}+\sum_{n=k+1}^{N} \underline{f}^{n}}
$$

$$
\begin{gathered}
\equiv \frac{\sum_{n=1}^{L} \bar{f}^{n} \underline{y}^{n}+\sum_{n=L+1}^{N} \underline{f}^{n} \underline{y}^{n}}{\sum_{n=1}^{L} \bar{f}^{n}+\sum_{n=L+1}^{N} \underline{f}^{n}} \\
y_{r}=\min _{k \in[1, N-1]} \frac{\sum_{n=1}^{k} \underline{f}^{n} \bar{y}^{n}+\sum_{n=k+1}^{N} \bar{f}^{n} \bar{y}^{n}}{\sum_{n=1}^{k} \underline{f}^{n}+\sum_{n=k+1}^{N} \bar{f}^{n}} \\
\frac{\sum_{n=1}^{R} \underline{f}^{n} \bar{y}^{n}+\sum_{n=R+1}^{N} \bar{f}^{n} \bar{y}^{n}}{\sum_{n=1}^{R} \underline{f}^{n}+\sum_{n=R+1}^{N} \bar{f}^{n}}
\end{gathered}
$$

From the type-reducer, we obtain an interval set $Y_{C o s}$, to defuzzify it we use the average of $y_{l}$ and $y_{r}$, so the defuzzified output of an interval singleton type-2 FLS is

$$
y(x)=\frac{y_{l}+y_{r}}{2}
$$

Above expression produces a final crisp value output to the type-2 fuzzy system.

\section{PROPOSED MODEL}

Based on the IT2FLS mentioned in the previous section, which is employ to solve the opportunistic spectrum access problem in CR networks. This technique provides expert knowledge for selecting the best suitable SU to access the unutilized band. The rule based decision making scheme consider three parameters of SU as antecedents such as spectrum utilization efficiency, degree of mobility and distance to the primary user. By using the rule-based IT2FLS, we combine the above three descriptors to determine optimal solutions to allocate spectrum opportunistically. We observe that various users will perceive different accessible spectrum and using spectrum efficiently, is the main purpose of the opportunistic spectrum access schemes. Therefore, spectrum utilization efficiency is considered in our fuzzy inference design, which is defined as the ratio between the spectrum band which will be utilized by the SU and the available band

$$
\eta_{s}=\frac{B W_{s}}{B W_{a}} \times 100 \%
$$

Where, $B W_{s}$ and $B W_{a}$ are the spectrum band which will be used by the secondary and the accessible band, respectively. Velocity or Mobility of the SU is the important factor in our design. When the SU is moving at a velocity $V \mathrm{~m} / \mathrm{s}$, it causes the Doppler Effect.

$$
f_{D}=\frac{v \cos \theta}{c} \times f_{c}
$$

Where, $f_{D}$ is Doppler shift frequency, $\theta$ is the arrival angle of the received signal, $\mathrm{c}$ is the wave velocity and $f_{c}$ is the carrier frequency. If the velocity is grater then there can be degradation in quality of service due to unavailability of the channel. The mobility of SU can be determined from the following relationship

$$
f=f_{m} \cos \varphi
$$


Where, $f_{m}=v / \lambda$ as carrier frequency, $v$ is the mobility, $\varphi$ is the angle and $\lambda$ is the carrier wavelength. Mobility can reduce ability of detecting signal from the PU. If the SU is not capable of detecting the primary signal, it will imperfectly determine that the spectrum is unused; thereby leading to potential interference to adjacent users, i.e., the signal transmitted by the SU will interfere with the signal that the PU is trying to decode. This situation is often called as the hidden node problem. In addition, Distance of the SU is another important factor because $\mathrm{SU}$ at a closer distance should be given priority to access spectrum, which depends upon the signal to noise ratio as an alternative for distance, at the $\mathrm{SU}$ is given by

$$
\gamma_{s}=10 \log \left(P_{1} g(R) / \sigma^{2}\right)
$$

Assume the PU at the distance R from the SU transmits signal at power P1 and the power gain between the PU and SU, $g(R)$ is a continuous, positive, strictly decreasing function of $\mathrm{R}$ described in the interval 0 to $\infty$. In IT2 fuzzy inference engine, three antecedent propositions can be expressed in three fuzzy partitions such as Low/Near, Moderate and High/Far. The result of decision making i.e. the possibility that the $\mathrm{SU}$ is selected to utilize the spectrum, which is divided in to five levels which are Very Low, Low, Medium, High and Very High. We employ trapezoidal and triangular membership functions (MFs) to represents input as well as output parameters of decision making structure. MFs are shown in Fig. 5, 6 and 7, since we have three antecedents and three fuzzy subsets, we need to set up 27 rules for this fuzzy system. Then, we design rules, which will be depending on various operating conditions and human knowledge as illustrated in Table 1. The rule structure is described as follows

$R_{l}$ : IF Spectrum utilization efficiency of $S U\left(x_{1}\right)$ is $A_{1 l}$ and its degree of mobility $\left(x_{2}\right)$ is $A_{2 l}$ and its distance to the primary user(x3) is $A_{3 l}$ THEN the possibility (y) that $S U$ is chosen to access the available spectrum is $B_{l}$

Table.1. Rule base for opportunistic spectrum access problem in CRs

\begin{tabular}{|l|l|l|l|l|}
\hline Rule & $\begin{array}{c}\text { Antecedent } \\
(\mathbf{1})\end{array}$ & $\begin{array}{c}\text { Antecedent } \\
(\mathbf{2})\end{array}$ & $\begin{array}{c}\text { Antecedent } \\
(\mathbf{3})\end{array}$ & Consequence \\
\hline 01 & Low & Low & Near & Very Low \\
\hline 02 & Low & Low & Moderate & Low \\
\hline 03 & Low & Low & Far & Low \\
\hline 04 & Low & Moderate & Near & Very Low \\
\hline 05 & Low & Moderate & Moderate & Low \\
\hline 06 & Low & Moderate & Far & Medium \\
\hline 07 & Low & High & Near & Very Low \\
\hline 08 & Low & High & Moderate & Low \\
\hline 09 & Low & High & Far & Medium \\
\hline 10 & Moderate & Low & Near & Very Low \\
\hline 11 & Moderate & Low & Moderate & Medium \\
\hline 12 & Moderate & Low & Far & High \\
\hline 13 & Moderate & Moderate & Near & Very Low \\
\hline 14 & Moderate & Moderate & Moderate & Medium \\
\hline 15 & Moderate & Moderate & Far & High \\
\hline 16 & Moderate & High & Near & Very Low \\
\hline 17 & Moderate & High & Moderate & Low \\
\hline 18 & Moderate & High & Far & High \\
\hline 19 & High & Low & Near & Low \\
\hline 20 & High & Low & Moderate & High \\
\hline 21 & High & Low & Far & Very High \\
\hline
\end{tabular}

\begin{tabular}{|l|l|l|l|l|}
\hline 22 & High & Moderate & Near & Low \\
\hline 23 & High & Moderate & Moderate & High \\
\hline 24 & High & Moderate & Far & Very High \\
\hline 25 & High & High & Near & Very Low \\
\hline 26 & High & High & Moderate & High \\
\hline 27 & High & High & Far & High \\
\hline
\end{tabular}

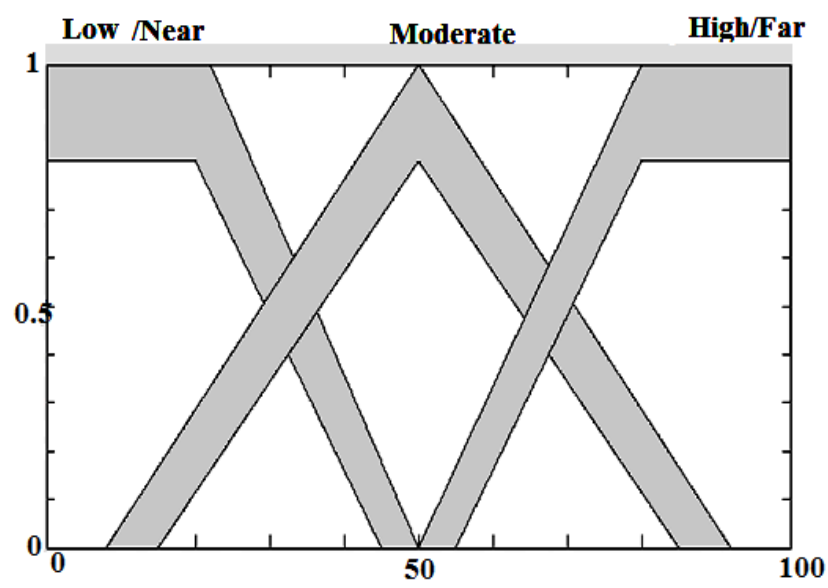

Fig 5: Input Membership Functions of spectrum utilization efficiency in IT2FLS

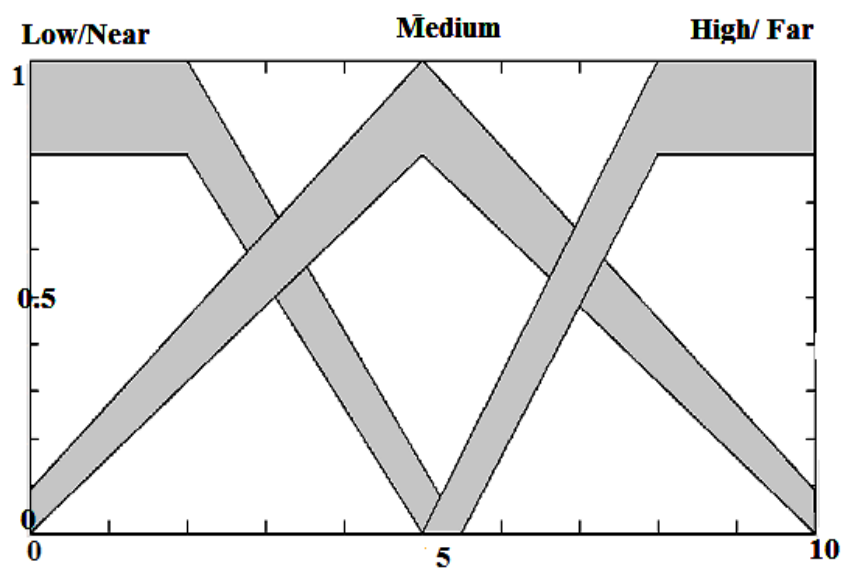

Fig 6: Input Membership Functions of Degree of mobility and distance between PU \& SU in IT2FLS

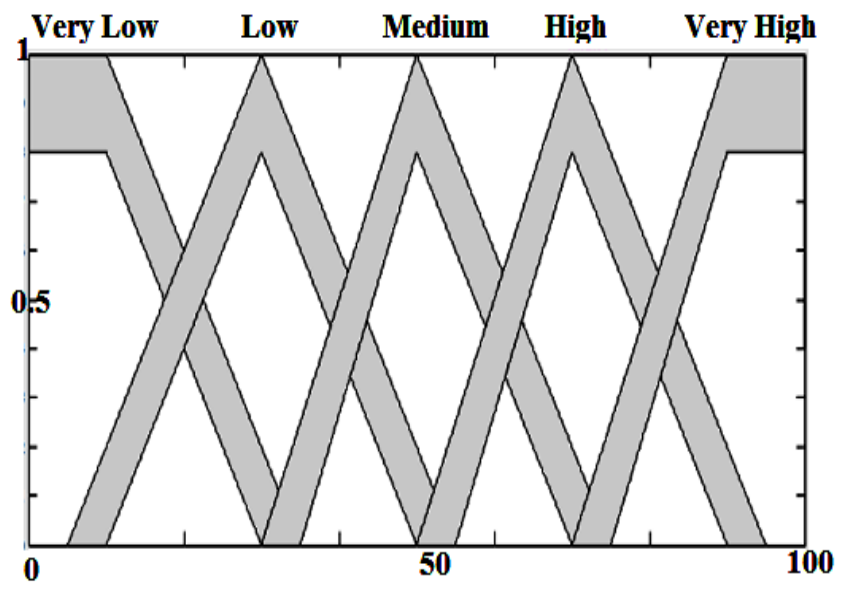

Fig 7: Output Membership Functions expressed in IT2FLS 


\section{SIMULATION RESULT}

To evaluate the response of IT2FLS in CRs, we randomly generated normalize sequence values of three descriptors as spectrum utilization efficiency of each SU was a random value in the interval [ $\left[\begin{array}{ll}0 & 100\end{array}\right]$ and its mobility degree in [0 10] with third parameter as the distances to the PU were normalized to [0 10]. The output of fuzzy decision making, i.e. the possibility that a SU was selected to access the available spectrum was computed and Fig. 8 represents the opportunistic spectrum access decision surface for the cognitive user. The output responses of IT2FLS decision making with respect to each input parameters of CR are shown through Fig. 9 The efficiency of the decision making process in CR is analyzed with help of IT2FLS and T1FLS approach.

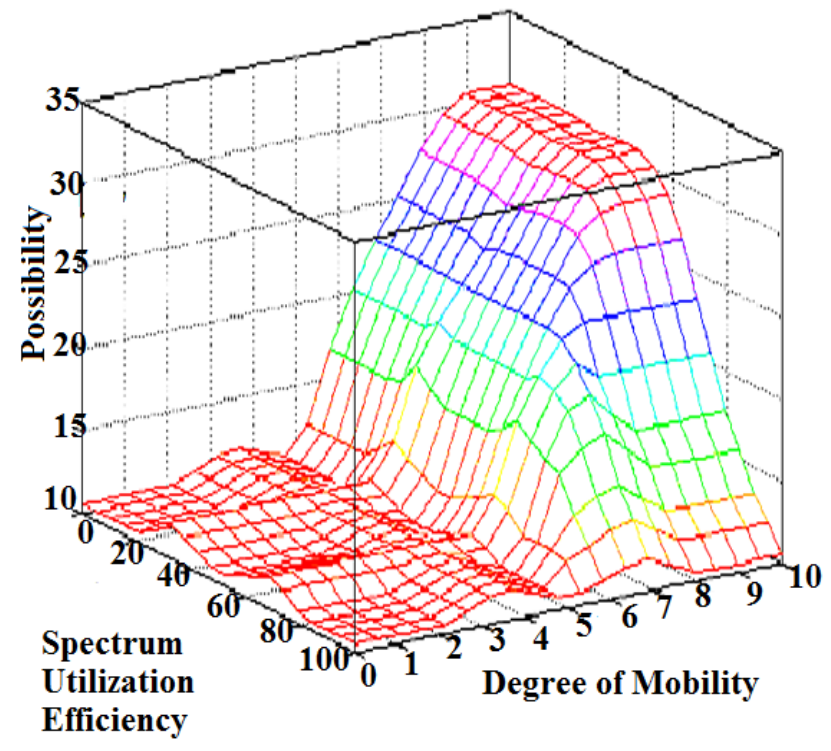

Fig 8 (a): Distance to PU fixed

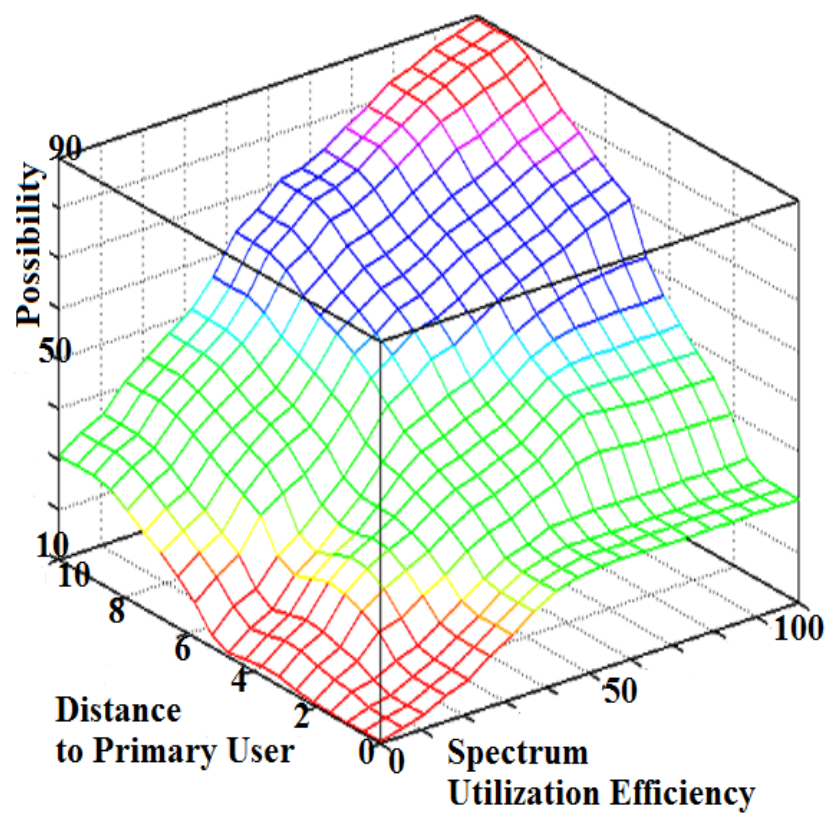

Fig 8(b): Degree of mobility is fixed

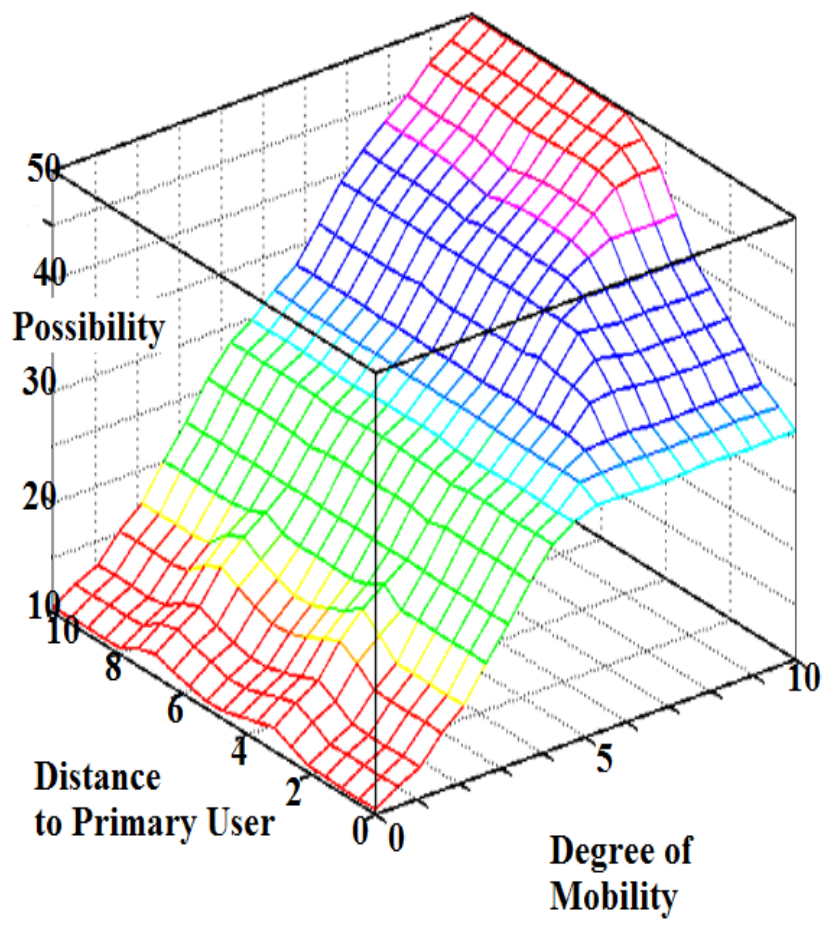

Fig 8(c): Spectrum utilization efficiency is fixed

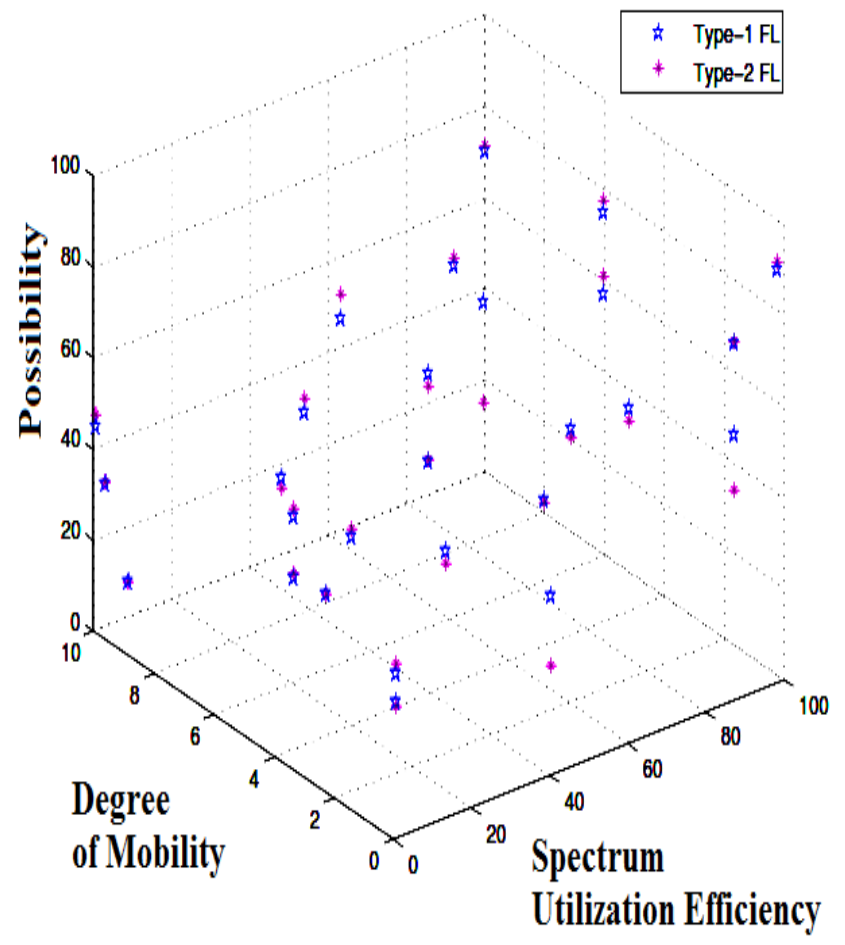

Fig 9 (a): The overall output response of fuzzy inference engine in CR, with a fixed distance to the PU 


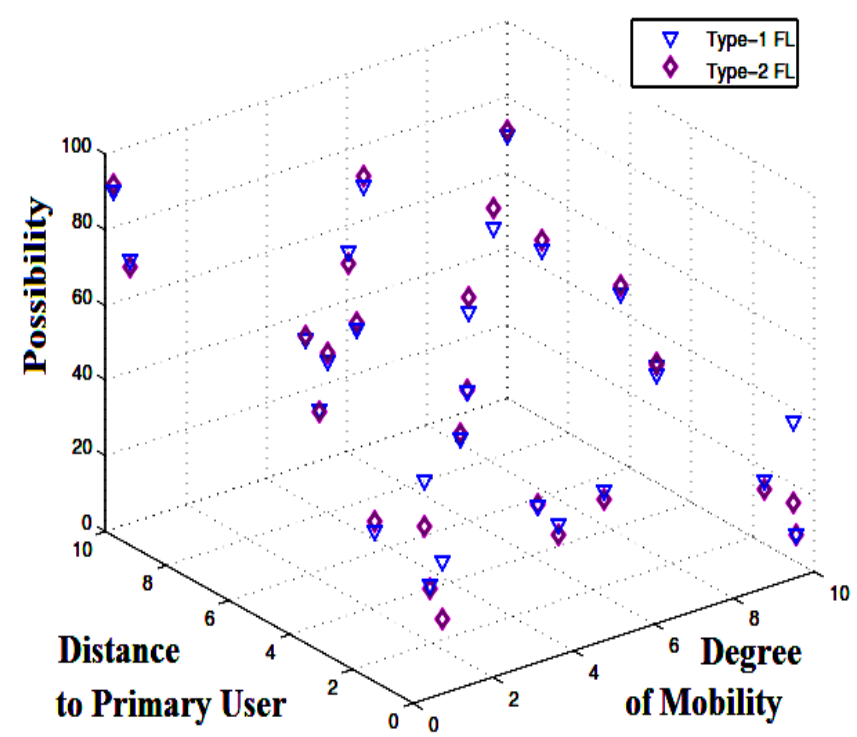

Fig 9(b): Degree of mobility is fixed

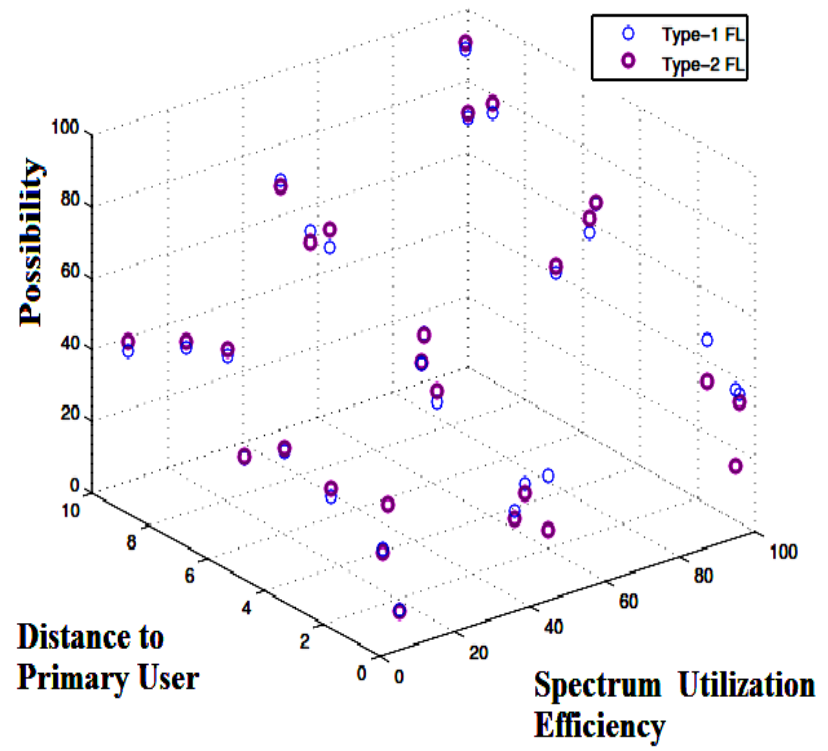

Fig 9 (c): Spectrum utilization efficiency is fixed

\section{CONCLUSION}

An improved approach is proposed using the IT2FLS for the opportunistic spectrum access in CR networks. The selection SU for spectrum access is based on three descriptors, i.e., spectrum utilization efficiency of the SU, its degree of mobility, and its distance to the PU. A mamdani type fuzzy inference engine has been employed, based on expert knowledge base an acceptable decision can be done. As a result, we represent the opportunistic spectrum access decision surface. The simulation results were compared between T1FLS and IT2FLS based decision making scheme for analyzing improvement in sensing accuracy due to uncertain MFs. The T2FLS has intensive computation due to the heavy computational load at the step of the type-reducing process, which can be simplified with help of IT2FLS in decision making process in CR. Therefore, proposed technique exhibit higher probability of detection and low false alarms in cognitive user. From simulation results, it is clear that, the chance of decision increases if the distance between licensed and unlicensed user is low and the velocity of the SU is more.

\section{ACKNOWLEDGMENTS}

Our thanks to the experts who have contributed towards development of the type-2 fuzzy logic system.

\section{REFERENCES}

[1] J. Mitola and G.Q. Maguire, 1999 "Cognitive radio: making software radios more personal”, IEEE Personal Communications, Vol. 6, pp.13-18.

[2] Simon Haykin, 2005 "Cognitive Radio: BrainEmpowered Wireless Communications", IEEE Journal on Selected Areas in Communications, Vol. 23, No. 2, pp 201-220.

[3] Ming-Ying Hsiao, Tzuu-Hseng S. Li , J. Z. Lee, C. H. Chao and S. H. Tsai, 2008 "Design of interval type-2 fuzzy sliding-mode controller", Information Sciences, Vol. 178, pp 1696-1716.

[4] Sumit Ghosh, Qutaiba Razouqi, H. Jerry Schumacher, and Aivars Celmins, 1998. "A Survey of Recent Advances in Fuzzy Logic in Telecommunications Networks and New Challenges", IEEE Transactions on Fuzzy Systems, Vol. 6, No. 3, pp 443-447.

[5] Nicola Baldo and Michele Zorzi, 2009 "Cognitive network access using fuzzy decision making", IEEE Transactions on Wireless Communications, Vol. 8, No. 7, pp-3523-3535.

[6] Marja Matinmikko, Tapio Rauma, Miia Mustonen, Ilkka Harjula, Heli Sarvanko and Aarne Mammela 2009 "Application of fuzzy logic to cognitive radio systems", IEICE Trans. Communication, Vol. E-92-B, No. 12, pp3572-3580.

[7] Prabhjot Kaur, Moin Uddin, and Arun Khosla. 2010 "Fuzzy Based Adaptive Bandwidth Allocation Scheme in Cognitive Radio Networks", International Conference on ICT and Knowledge Engineering, pp 41-45.

[8] Hong-Sam T. Le and Hung D. Ly. 2011, "Opportunistic Spectrum Access Using Fuzzy Logic for Cognitive Radio Networks", International Journal of Wireless Information Networks, Vol. 18, No. 3, pp 171-178.

[9] F. Koroupi, H. Salehinejad, and S. Talebi, 2013. "Spectrum Assignment In Cognitive Radio Networks Using Fuzzy Logic Empowered Ants", Iranian Journal of Fuzzy Systems, Vol. 10, No. 6, pp. 1-19.

[10] Mansi Subhedar, Gajanan Birajdar, 2013. "Comparison of mamdani and sugeno inference systems for dynamic spectrum allocation in cognitive radio networks", Wireless Personal Communication, Vol. 71, pp. 805-819. 